\title{
CHAIN CONDITIONS IN THE DISTRIBUTIVE FREE PRODUCT OF LATTICES
}

\author{
BY \\ G. GRÄTZER AND H. LAKSER( $\left.{ }^{1}\right)$
}

1. Introduction. A typical result of this paper is the following. Let $L_{i}, i \in I$ be distributive lattices satisfying the countable chain condition. Then the free product $L$ of these lattices also satisfies the countable chain condition.

To be able to state the general result we need some notations.

Let $\mathfrak{m}$ be an infinite cardinal. A poset (partially ordered set) $P$ is said to satisfy the $\mathfrak{m}$-chain condition if every chain in $P$ has cardinality $<\mathfrak{m}$. (Thus the countable chain condition is the $\boldsymbol{X}_{1}$-chain condition.) Given a category of lattices that admits free products, a cardinal $\mathfrak{n}>1$, and an infinite cardinal $\mathfrak{m}$ we define condition $P(\mathfrak{m}, \mathfrak{n})$ :

Definition 1. A category $K$ of lattices is said to satisfy $P(\mathfrak{m}, \mathfrak{n})$ if, given a family $\left(L_{i} \mid i \in I\right)$ of lattices in the category such that:

(i) $|I|=\mathfrak{n}$,

(ii) for each $i \in I, L_{i}$ satisfies the $m$-chain condition,

then the free product in $K$ of the $\left(L_{i} \mid i \in I\right)$ satisfies the m-chain condition.

Our main result is

THEOREM. For each of the category of distributive lattices, the category of distributive lattices with 0,1 , and the category of Boolean algebras:

(a) for $\mathfrak{n} \geqq \boldsymbol{\aleph}_{0}, \boldsymbol{P}(\mathfrak{m}, \mathfrak{n})$ holds if and only if $\mathfrak{m}$ is a regular cardinal greater than $\boldsymbol{\aleph}_{0}$;

(b) for $1<\mathfrak{n}<\mathbb{X}_{0}, P(\mathfrak{m}, \mathfrak{n})$ holds if and only if $\mathfrak{m}$ is either regular or cofinal $\left({ }^{2}\right)$ with $\omega$.

There is a very intimate connection between free products in the three categories mentioned in the theorem. We note first that the free product of a family of Boolean algebras is identical with their free product as distributive lattices with 0,1 . We also observe that given any distributive lattice we can adjoin a 0 and 1 in the obvious way; in this manner homomorphisms of distributive lattices yield homomorphisms preserving 0,1 . The following fact is evident.

(*) Let $\left(L_{i} \mid i \in I\right)$ be a family of distributive lattices. For each $i \in I$, let $L_{i}^{*}$ be the result of adjoining 0 and 1 to $L_{i}$. Let $L^{*}$ be the $\{0,1\}$-distributive free product of $\left(L_{i}^{*} \mid i \in I\right)$. Then $L=L^{*}-\{0,1\}$ is a distributive lattice and is the free product of the family $\left(L_{i} \mid i \in I\right)$ in the category of distributive lattices.

Presented to the Society, January 24, 1969; received by the editors February 5, 1969.

( ${ }^{1}$ ) This research was supported by the National Research Council of Canada and by the Canadian Mathematical Congress.

$\left.{ }^{2}\right) \mathfrak{m}$ is cofinal with $\omega$, if $\mathfrak{m}=\sum\left(\mathfrak{m}_{i} \mid i \in I\right)$, with $\mathfrak{m}_{i}<\mathfrak{m},|I|=\mathfrak{\aleph}_{0}$. 
In view of the above discussion all of the positive results in this paper will be proved for the category of distributive lattices with 0,1 ; we need only observe that adjoining 0 and 1 does not alter the cardinality of any infinite chain. All of the examples needed to establish our negative results, on the other hand, will be Boolean algebras.

All of our positive results are applicable, with the obvious changes, to the category of distributive lattices (and, indeed, to the intermediate categories preserving 0 or preserving 1 ) and we leave to the reader the task of formulating the results in these contexts.

Certain trivial cases of our result, namely the proof of the $\boldsymbol{\aleph}_{1}$-chain condition for free distributive lattices and free Boolean algebras, appeared in [3] and [5]. A very elegant proof of this result, which does not seem to be applicable to our situation, was presented by F. Galvin and B. Jónsson [1].

2. Preliminaries on the free product. The usual construction of distributive free products of distributive lattices is by way of the Stone representation theorem. (See e.g. [4].) A disadvantage of this approach is its reliance on the Axiom of Choice. In our approach to the free product we dispense with this requirement.

A straightforward extension of the results of $\$ \$ 28$ and 29 of [2] is the following:

THEOREM 1. Let $K$ be an equational class of algebras and let $\left(\mathfrak{A}_{i} \mid i \in I\right)$ be a family of algebras in $K$. A necessary and sufficient condition for the existence of the free product $\left({ }^{3}\right)$ in $K$ of the family $\left(\mathfrak{U}_{i} \mid i \in I\right)$ is:

Given any finite $I^{\prime} \subseteq I$, finitely generated subalgebras $\mathfrak{B}_{i}$ of $\mathfrak{A}_{i}$ for $i \in I^{\prime}$, any $j \in I^{\prime}$, and distinct $a, b \in B_{j}$, then there is an algebra $\mathfrak{B}$ in $K$ and homomorphisms $f_{i}: \mathfrak{B}_{\mathfrak{i}} \rightarrow \mathfrak{B}$, $i \in I^{\prime}$, such that $f_{j}(a) \neq f_{j}(b)$.

COROLlARY 1. In an equational class $K$ of algebras where free products of finitely many finitely generated algebras always exist, free products of arbitrarily many arbitrary algebras exist.

Since finitely generated distributive lattices are finite the existence of free products of finitely generated distributive lattices is independent of the Axiom of Choice.

COROLlaRy 2. Free products exist in the category of distributive lattices with 0,1 and with more than one element.

It should be stressed that neither Theorem 1 nor its corollaries use the Axiom of Choice, nor the weaker Prime Ideal Theorem.

Before characterizing the free product of distributive lattices we present a wellknown preliminary lemma.

$\left.{ }^{3}\right)$ We use the algebraic definition of free products as in [2], that is the $\mathfrak{A}_{\mathfrak{l}}$ are represented by isomorphic copies in the free product. 
Lemma 1. Let $L$ be a distributive lattice with 0,1 and let $x, y \in L$. If $x \neq y$ then there is a distributive lattice $M$ with 0,1 and a lattice homomorphism $\varphi: L \rightarrow M$ such that $\varphi(x)=1, \varphi(y)=0$.

Proof. We define a relation $\Theta$ on $L$ :

$$
a \equiv b(\Theta) \text { if and only if }(a \vee y) \wedge x=(b \vee y) \wedge x .
$$

It is clear that $\Theta$ is an equivalence relation, and a simple calculation establishes that $\Theta$ has the substitution property for $\vee$ and $\wedge$; consequently $\Theta$ is a congruence relation on $L$. Since $a \vee y \equiv a(\Theta)$ and $a \wedge x \equiv a(\Theta)$ for all $a \in L$, the quotient lattice $L / \Theta$ is a suitable candidate for $M$.

THEOREM 2. Let $L$ be a distributive lattice with 0,1 and let $\left(L_{i} \mid i \in I\right)$ be a family of $\{0,1\}$-sublattices of $L$ that generates $L$. Then $L$ is the $\{0,1\}$-free product of the $\left(L_{i} \mid i \in I\right)$ if and only if, given finite $I_{1}, I_{2} \subseteq I$ and elements $x_{i} \in L_{i}, i \in I_{1}, y_{j} \in L_{j}$, $j \in I_{2}$, the relation

$$
\bigwedge\left(x_{i} \mid i \in I_{1}\right) \leqq \bigvee\left(y_{j} \mid j \in I_{2}\right)
$$

implies that there is an $i \in I_{1} \cap I_{2}$ such that $x_{i} \leqq y_{i}$.

Proof. We first prove the necessity of the condition. Let $L$ be the free product of the $\left(L_{i} \mid i \in I\right)$. Assume that the condition fails to hold; that is, $x_{i} y_{i}$ for each $i \in I_{1} \cap I_{2}$. We may assume that none of the $x_{i}$ is 0 and none of the $y_{j}$ is 1 . We construct a family $\left(M_{i} \mid i \in I\right)$ of distributive lattices with 0,1 :

For each $i \in I_{1} \cap I_{2}$ let $M_{i}$ be the lattice constructed in Lemma 1. Thus, for each $i \in I_{1} \cap I_{2}$, there is a homomorphism $\varphi_{i}: L_{i} \rightarrow M_{i}$ mapping $x_{i}$ to 1 and $y_{i}$ to 0 .

For $i \in I_{1}-I_{2}$ we use Lemma 1 to get a lattice $M_{i}$ and a homomorphism $\varphi_{i}: L_{i} \rightarrow M_{i}$ such that $\varphi_{i}\left(x_{i}\right)=1$.

Similarly, for $i \in I_{2}-I_{1}, \varphi_{i}: L_{i} \rightarrow M_{i}$ maps $y_{i}$ to 0 .

For $i \in I-\left(I_{1} \cup I_{2}\right)$ let $M_{i}=L_{i}$ and let $\varphi_{i}$ be the identity.

Let $M$ be the $\{0,1\}$-distributive free product of the $\left(M_{i} \mid i \in I\right)$. Then there is a homomorphism $f: L \rightarrow M$ extending the $\varphi_{i}$. Consequently

$$
f\left(x_{i}\right)=1, \quad i \in I_{1}, \quad \text { and } f\left(y_{j}\right)=0, \quad j \in I_{2} ;
$$

thus

$$
f\left(\bigwedge\left(x_{i} \mid i \in I_{1}\right)\right)=1>0=f\left(\bigvee\left(y_{j} \mid j \in I_{2}\right)\right),
$$

contradicting the inequality

$$
\bigwedge\left(x_{i} \mid i \in I_{1}\right) \leqq \bigvee\left(y_{j} \mid j \in I_{2}\right) .
$$

Thus the condition is necessary.

Now let the condition hold, and let $L^{*}$ be the free product of the $\left(L_{i} \mid i \in I\right)$. Then there is a homomorphism $f$ of $L^{*}$ onto $L$ extending the embedding into $L$ of 
each $L_{i}$. To show that $f$ is an isomorphism it suffices to show, given $a, b \in L^{*}$, that $f(a) \leqq f(b)$ implies $a \leqq b$. Using distributivity we may write

$$
\begin{aligned}
& a=\bigwedge\left(x_{i} \mid i \in I_{1}\right) \vee \cdots \vee \wedge\left(x_{i} \mid i \in I_{m}\right), \\
& b=\bigvee\left(y_{j} \mid j \in J_{1}\right) \wedge \cdots \wedge \bigvee\left(y_{j} \mid j \in J_{n}\right),
\end{aligned}
$$

where the $I_{p}, J_{p}$ are finite subsets of $I$. If $f(a) \leqq f(b)$ then for each $p \leqq m, q \leqq n$

$$
\bigwedge\left(x_{i} \mid i \in I_{p}\right) \leqq \bigvee\left(y_{j} \mid j \in J_{q}\right) \text { in } L .
$$

Thus for each $p \leqq m, q \leqq n$ there is an $i \in I_{p} \cap J_{q}$ such that $x_{i} \leqq y_{i}$ in $L_{i}$. Thus, for all $p, q$,

\section{Consequently $a \leqq b$.}

$$
\bigwedge\left(x_{i} \mid i \in I_{p}\right) \leqq \bigvee\left(y_{j} \mid j \in J_{q}\right) \text { in } L^{*}
$$

Thus the sufficiency of the condition is established and so the theorem is proved.

Remark. Had we been willing to use the Axiom of Choice the lattice $M$ of Lemma 1 could have been taken as the two-element lattice and so the "necessary" part of the proof of Theorem 2 could have been simplified; the lattice $M$ in that part would have been the two-element lattice.

3. The word problem. Let $L$ be the distributive $\{0,1\}$-free product of the family $\left(L_{i} \mid i \in I\right)$ and let

$$
Q=\bigcup\left(L_{i}-\{0,1\} \mid i \in I\right) \cup\{0,1\} .
$$

We identify $0 \in Q$ with $0 \in L_{i}$ for each $i \in I$, and similarly for $1 \in Q$. A finite nonempty subset $X \subseteq Q$ is said to be reduced if $\left|X \cap L_{i}\right| \leqq 1$ for all $i \in I$. It should be noted that if $X$ is reduced and $0 \in X$ then $X=\{0\}$, and dually. If $X \subseteq Q$ is finite and nonempty we can define a reduced subset $X^{\wedge}$ of $Q$, the $\wedge$-reduct of $X$, by the conditions:

(i) if $I^{\prime}=\left\{i \in I \mid X \cap L_{i} \neq \varnothing\right\}$ then

$$
X^{\wedge}=\left\{\bigwedge\left(X \cap L_{i}\right) \mid i \in I^{\prime}\right\}
$$

provided that $\bigwedge\left(X \cap L_{i}\right) \neq 0$ for all $i \in I^{\prime}$;

(ii) if there is an $i \in I^{\prime}$ such that $\bigwedge\left(X \cap L_{i}\right)=0$ then

$$
X^{\wedge}=\{0\} .
$$

The $\bigvee$-reduct of $X$, denoted $X^{\vee}$, is defined in the dual manner. We note that, in $L$, $\bigwedge X=\wedge\left(X^{\wedge}\right)$ and $\bigvee X=\bigvee\left(X^{\vee}\right)$

We observe that, since $L$ is a distributive lattice generated by $Q$, each element $a$ of $L$ can be written (in a nonunique manner) as $a=\bigwedge(\bigvee X \mid X \in J)$ where $J$ is a finite family of finite reduced subsets of $Q$. Conversely, any such family $J$ yields an element $\bigwedge(\bigvee X \mid X \in J)$ of $L$.

Definition 2. A finite family $J$ of finite reduced subsets of $Q$ is said to be a $\bigvee$-representation of $a \in L$ if

$$
a=\bigvee(\bigwedge X \mid X \in J)
$$


The family $J$ is said to be a $\bigwedge$-representation of $a \in L$ if

$$
a=\wedge(\bigvee X \mid X \in J)
$$

Given a $\wedge$-representation $J$ of an element $a \in L$ we can write, using distributivity,

$$
a=\bigvee(\bigwedge(F(J)) \mid F \in \mathscr{C}(J))
$$

$\mathscr{C}(J)$ denotes the set of choice functions on $J$, that is, the set of functions $F: J \rightarrow \cup J$ such that $F(X) \in X$ for each $X \in J$. By our previous discussion we find that $a=\bigvee\left(\bigwedge\left(F(J)^{\wedge}\right) \mid F \in \mathscr{C}(J)\right)$. Since the set $\mathscr{C}(J)$ is finite we can consider a subset $\mathscr{C}_{\text {red }}(J) \subseteq \mathscr{C}(J)$, the set of reduced choice functions such that the set

$$
\left\{\bigwedge\left(F(J)^{\wedge}\right) \mid F \in \mathscr{C}_{\text {red }}(J)\right\}
$$

is the set of all maximal elements of the $\operatorname{set}\left({ }^{4}\right)$

$$
\left\{\bigwedge\left(F(J)^{\wedge}\right) \mid F \in \mathscr{C}(J)\right\} .
$$

Thus the family $\left\{F(J)^{\wedge} \mid F \in \mathscr{C}_{\text {red }}(J)\right\}$ is a $\bigvee$-representation of $a$; it is said to be a normal $\bigvee$-representation of $a$. There is, of course, a dual normal $\wedge$-representation of $a$.

Lemma 2. If $X, Y$ are finite reduced subsets of $Q$ then $\wedge X \leqq \wedge Y$ in $L$ if and only if for each $y \in Y$ there is an $x \in X$ such that $x \leqq y$ (and thus $x, y$ are in the same $L_{i}$ ).

Proof. If for each $y \in Y$ there is an $x \in X$ such that $x \leqq y$ then clearly $\bigwedge X \leqq \bigwedge Y$. The converse follows by Theorem 2. Let $\bigwedge X \leqq \bigwedge Y$ and let $y \in Y$. Since $X$ is reduced there is a finite $I_{1} \subseteq I$ such that $X=\left\{x_{i} \mid i \in I_{1}\right\}$ where $x_{i} \in L_{i}$. Thus $\bigwedge\left(x_{i} \mid i \in I_{1}\right) \leqq y$ and so there is an $i \in I_{1}$ such that $x_{i}, y \in L_{i}$ and $x_{i} \leqq y$.

THEOREM 3. (i) Each $a \in L$ has a normal $\bigvee$-representation. (ii) Let $a, b \in L$ and let $J_{1}$ be $a \bigvee$-representation of $a$ and $J_{2}$ a normal $\bigvee$-representation of $b$. Then $a \leqq b$ if and only if the following condition holds:

For each $X \in J_{1}$ there is a $Y \in J_{2}$ such that $\bigwedge X \leqq \bigwedge Y$, that is, for each $y \in Y$ there is an $x \in X$ such that $x \leqq y$.

Proof. Since

$$
\begin{aligned}
& a=\bigvee\left(\bigwedge X \mid X \in J_{1}\right), \\
& b=\bigvee\left(\bigwedge Y \mid Y \in J_{2}\right),
\end{aligned}
$$

the condition is clearly sufficient for $a \leqq b$.

Now let $a \leqq b$ and let $K$ be a $\bigwedge$-representation of $b$ such that

$$
J_{2}=\left\{F(K)^{\wedge} \mid F \in \mathscr{C}_{\text {red }}(K)\right\} .
$$

${ }^{(4)}$ We require that there be no repetitions; that is, if $F, G \in C_{\text {red }}(J)$ and $\wedge\left(F(J)^{\wedge}\right)=\wedge\left(G(J)^{\wedge}\right)$ then $F=G$. Thus $C_{\text {red }}(J)$ is not determined uniquely by $J$. This, however, causes no difficulty; see the corollary of Theorem 3 . 
Thus

$$
\bigvee\left(\bigwedge X \mid X \in J_{1}\right) \leqq \wedge(\bigvee Z \mid Z \in K)
$$

Thus if $X \in J_{1}$ then $\bigwedge X \leqq \bigvee Z$ for each $Z \in K$. Since both $X$ and each $Z$ are reduced we conclude, by Theorem 2 , that for each $Z \in K$ there is an element $G(Z) \in Z$ such that $\bigwedge X \leqq G(Z)$. Thus

$$
\bigwedge X \leqq \bigwedge(G(Z) \mid Z \in K)=\bigwedge\left(G(K)^{\wedge}\right)
$$

where, clearly, $G \in \mathscr{C}(K)$. By the definition of $\mathscr{C}_{\text {red }}(K)$ there is an $F \in \mathscr{C}_{\text {red }}(K)$ such that $\bigwedge\left(G(K)^{\wedge}\right) \leqq \bigwedge\left(F(K)^{\wedge}\right)$.

The rest of the condition follows by Lemma 2 . Thus the theorem is proved.

Since in a normal $\bigvee$-representation $J$ the elements of $\{\bigwedge X \mid X \in J\}$ are mutually incomparable we conclude that

COROLLARY. The normal $\bigvee$-representation of any element of $L$ is uniquely defined.

EXAMPLE. Let $L$ be the free product of $L_{0}$ and $L_{1}, a_{0}, b_{0} \in L_{0}, a_{1}, b_{1} \in L_{1}$; let $x \in L$ be defined by

$$
x=\left(a_{0} \wedge a_{1}\right) \vee\left(b_{0} \wedge b_{1}\right) .
$$

Assume that $a_{i}$ is incomparable to $b_{i}, i=0,1$, and let $c_{i}=a_{i} \wedge b_{i}, d_{i}=a_{i} \vee b_{i}$, $i=0,1$. Then the normal $\bigvee$-representation $J$ represents $x=\bigvee J$ as follows:

$$
x=\left(a_{0} \wedge a_{1}\right) \vee\left(c_{0} \wedge d_{1}\right) \vee\left(d_{0} \wedge c_{1}\right) \vee\left(b_{0} \wedge b_{1}\right)
$$

and the normal $\bigwedge$-representation yields:

$$
x=d_{0} \wedge\left(a_{0} \vee b_{1}\right) \wedge\left(a_{1} \vee b_{0}\right) \wedge d_{1} .
$$

We do not know whether $\bigvee$-, and $\bigwedge$-representations contain the same number of factors.

4. The chain condition for regular cardinals. We first establish $P(\mathfrak{m}, \mathfrak{n})$ in the category of distributive lattices with 0,1 for a regular cardinal $m>\aleph_{0}$. Let $\left(L_{i} \mid i \in I\right)$ be a family of distributive lattices with 0,1 , let $|I|=\mathfrak{n}$, and let $L$ be their $\{0,1\}$ distributive free product. Let $\mathfrak{m}$ be regular and let $L_{\mathfrak{i}}$ satisfy the $\mathfrak{m}$-chain condition for each $i \in I$.

If $J$ is a $\bigvee$-representation of $a \in L$ we call $|J|$ the rank of the representation and $\sum(|X| \mid X \in J)$ the length of the representation.

If $H \subseteq L$, then a $\bigvee$-representation of $H, J(H)$, is a family $\left(J_{a} \mid a \in H\right)$, where $J_{a}$ is a $\bigvee$-representation of $a$. If $n$ is an integer and rank $J_{a}=n$ for each $a \in H$, then $J(H)$ is said to have rank $n$. A $\bigvee$-representation $J(H)$ of $H$ is said to be special if

(i) $a \in H, X, Y \in J_{a}$, and $\bigwedge X \leqq \bigwedge Y$ imply that $X=Y$;

(ii) $a, b \in H$ and $a \leqq b$ imply that for each $X \in J_{a}$ there is a $Y \in J_{b}$ such that $\bigwedge X \leqq \bigwedge Y$. 
Each $H \subseteq L$ has a special $\bigvee$-representation; by Theorem $3, J_{a}$ need only be chosen as a normal $\bigvee$-representation of $a$ for each $a \in H$. The example at the end of $\S 3$ can be used to show that a special $\bigvee$-representation need not be normal.

To establish $P(\mathfrak{m}, \mathfrak{n})$ we first present several lemmas.

LemmA 3. Let $C$ be a chain in $L$ that has $a \bigvee$-representation of rank 1 . Then $|C|<\mathfrak{m}$.

Proof. Let $J(C)$ be a $\bigvee$-representation of $C$ of rank 1. For each integer $n$ let

$$
C^{(n)}=\left\{a \in C \mid \text { length } J_{a}=n\right\} \text {. }
$$

For each $a \in C^{(n)}$ let $J_{a}=\left\{X_{a}\right\}$ and let

$$
I_{a}=\left\{i \in I \mid X_{a} \cap L_{i} \neq \varnothing\right\} .
$$

Since any pair, $a, b \in C^{(n)}$ are comparable and since $\left|I_{a}\right|=n=\left|I_{b}\right|$ we conclude, by Lemma 2, that $I_{a}=I_{b}$ for all $a, b \in C^{(n)}$. Set $I^{\prime}=I_{a}, a \in C^{(n)}$. For each $i \in I^{\prime}$ the set

$$
H_{i}=\left\{x \mid x \in X_{a} \cap L_{i} \text { and } a \in C^{(n)}\right\}
$$

is a chain in $L_{i}$ and so has cardinality $<\mathfrak{m}$. Since $C^{(n)}$ is isomorphic to a subset of $\Pi\left(H_{i} \mid i \in I^{\prime}\right)$ it follows that $\left|C^{(n)}\right|<\mathfrak{m}^{n}=\mathfrak{m}$. Since $\mathfrak{m}>\boldsymbol{\aleph}_{0}$ and regular and $C=\bigcup\left(C^{(n)} \mid n<\omega\right),|C|<\mathfrak{m}$, completing the proof of the lemma.

We now proceed by induction.

Let $C$ be a chain in $L$ with a special $\bigvee$-representation $J(C)$. For each $a, b \in C$, $a \leqq b$, we define a relation $\varphi_{a b} \subseteq J_{a} \times J_{b}$ with domain $J_{a}$ (the domain of a binary relation $r$ is the set of all $x$ for which there is a $y$ with $x r y$ ):

Then

$$
\varphi_{a b}=\left\{\langle X, Y\rangle \in J_{a} \times J_{b} \mid \wedge X \leqq \bigwedge Y\right\}
$$

(i) $\varphi_{a a}$ is equality (the diagonal of $J_{a} \times J_{a}$ );

(ii) if $a \leqq b \leqq c$ then $\left({ }^{5}\right) \varphi_{b c} \circ \varphi_{a b} \subseteq \varphi_{a c}$.

To proceed further we need the following generalization of the well-known fact that the inverse limit of a directed family of finite sets is nonempty.

LEMMA 4. Let $\Lambda$ be a directed set and let $\mathscr{H}=\left(H_{\lambda} \mid \lambda \in \Lambda\right)$ be a family of finite sets. For each pair $\lambda, \mu \in \Lambda$ such that $\lambda \leqq \mu$ let there be a relation $\varphi_{\lambda \mu} \subseteq H_{\lambda} \times H_{\mu}$ with domain $H_{\lambda}$ satisfying the two conditions:

(i) $\varphi_{\lambda \lambda}$ is equality for all $\lambda \in \Lambda$;

(ii) if $\lambda \leqq \mu \leqq \nu$ then $\varphi_{\mu \nu} \circ \varphi_{\lambda \mu} \subseteq \varphi_{\lambda \nu}$.

Then there is a family $\left(x_{\lambda} \in H_{\lambda} \mid \lambda \in \Lambda\right)$ such that $\left\langle x_{\lambda}, x_{\mu}\right\rangle \in \varphi_{\lambda \mu}$ if $\lambda \leqq \mu$.

The proof of this lemma is a trivial generalization of the usual topological proof, or of the algebraic proof presented in [2].

${ }^{(5)}$ This notation is the one consistent with writing mappings on the left: $\langle X, Y\rangle \in \varphi_{b c} \circ \varphi_{a b}$ if and only if there is a $Z \in J_{b}$ such that $\langle X, Z\rangle \in \varphi_{a b}$ and $\langle Z, Y\rangle \in \varphi_{b c}$. 
Now let $n$ be the smallest integer such that there is a chain $C \subseteq L$ where $|C| \geqq m$ and $C$ has a special $\bigvee$-representation $J(C)$ of rank $n$. Note that, by Lemma 3, $n>1$. Thus, by Lemma 4 , there is a family

$$
\mathscr{X}=\left(X_{a} \mid a \in C, X_{a} \in J_{a}, J_{a} \in J(C)\right)
$$

such that $\bigwedge X_{a} \leqq \bigwedge X_{b}$ whenever $a \leqq b$. Since $\mathscr{X}$ is a $\bigvee$-representation of rank 1 of a chain in $L,|\mathscr{X}|<\mathfrak{m}$ and so there is a subset $C^{\prime} \subseteq C$ such that

(i) $\left|C^{\prime}\right| \geqq \mathfrak{m}$ (since $\mathfrak{m}$ is regular);

(ii) if $a, b \in C^{\prime}$ and $X_{a}, X_{b} \in \mathscr{X}$ then $X_{a}=X_{b}$.

The family $\mathscr{J}=\left(J_{a}-\left\{X_{a}\right\} \mid a \in C^{\prime}\right)$ has cardinality $\geqq \mathfrak{m}$ and rank $n-1$. $\mathscr{J}$ is clearly a $\bigvee$-representation of some subset $H \subseteq L$, and clause (i) of the definition of a special representation is clear. Now let $a, b \in C^{\prime}, a \leqq b$, and let $X \in J_{a}-\left\{X_{a}\right\}$. Then there is a $Y \in J_{b}$ such that $\wedge X \leqq \bigwedge Y$. If $Y=X_{b}$ then, since $X_{a}=X_{b}$, $\bigwedge X \leqq \bigwedge X_{a}$, contradicting the fact that $J(C)$ is special. Thus $Y \in J_{b}-\left\{X_{b}\right\}$ and so $H$ is a chain with a special $\bigvee$-representation $\mathscr{J}$. However, rank $\mathscr{J}=n-1$ and $|H| \geqq \mathfrak{m}$, contradicting the minimality of $n$. Thus we conclude:

LEMMA 5. Let $n<\omega$. If $C$ is a chain in $L$ with a special $\bigvee$-representation of rank $n$ then $|C|<\mathrm{m}$.

Finally, let $C$ be a chain in $L$ and let $J(C)$ be a special $\bigvee$-representation of $C$. For each $n<\omega$ let

$$
C_{n}=\left\{a \in C \mid \operatorname{rank} J_{a}=n\right\} .
$$

Then $J\left(C_{n}\right)=\left(J_{a} \mid a \in C_{n}\right)$ is a special $\bigvee$-representation of $C_{n}$ of rank $n$. Thus $\left|C_{n}\right|<\mathfrak{m}$ and since $C=\bigcup\left(C_{n} \mid n<\omega\right)$ by the regularity of $\mathfrak{m}$ we get that $|C|<\mathfrak{m}$. Thus

THEOREM 4. In the category of distributive lattices with $0,1, P(\mathfrak{m}, \mathfrak{n})$ is true for all pairs of cardinals $\langle\mathfrak{m}, \mathfrak{n}\rangle$ such that $\mathfrak{m}>\boldsymbol{N}_{0}$ and regular.

5. Chain condition for singular cardinals. To discuss the case when $\mathfrak{m}$ is singular we need the concept of weak direct product of posets. Given a family $\left(P_{i} \mid i \in I\right)$ of posets with 0,1 the direct product is denoted by $\prod\left(P_{\mathfrak{i}} \mid i \in I\right)$. If $x \in \Pi\left(P_{\mathfrak{i}} \mid i \in I\right)$, the projection of $x$ on the factor $P_{i}$ is denoted $x_{i}$.

For each $x \in \prod\left(P_{\mathfrak{i}} \mid i \in I\right)$ set

$$
\begin{aligned}
& \operatorname{sp}_{0}(x)=\left\{i \in I \mid x_{i} \neq 0\right\} \\
& \operatorname{sp}_{1}(x)=\left\{i \in I \mid x_{i} \neq 1\right\}
\end{aligned}
$$

and define

$$
\prod_{0}\left(P_{i} \mid i \in I\right)=\left\{x \mid x \in \prod\left(P_{i} \mid i \in I\right) \text { and } \operatorname{sp}_{0}(x) \text { is finite }\right\}
$$

and

$$
\prod_{1}\left(P_{i} \mid i \in I\right)=\left\{x \mid x \in \prod\left(P_{i} \mid i \in I\right) \text { and } \operatorname{sp}_{1}(x) \text { is finite }\right\}
$$


The weak direct product of the family $\left(P_{i} \mid i \in I\right)$ is defined as

$$
\prod_{w}\left(P_{i} \mid i \in I\right)=\prod_{0}\left(P_{i} \mid i \in I\right) \cup \prod_{1}\left(P_{i} \mid i \in I\right) .
$$

Lemma 6. Let $m$ be an infinite cardinal not cofinal with $\omega$, and let $\left(P_{i} \mid i \in I\right)$ be a family of posets with 0,1 satisfying the m-chain condition. Then $\prod_{w}\left(P_{i} \mid i \in I\right)$ satisfies the $\mathrm{m}$-chain condition.

Proof. Let $C$ be a chain in $\prod_{w}\left(P_{i} \mid i \in I\right)$ and let

$$
C^{\prime}=C \cap \prod_{0}\left(P_{i} \mid i \in I\right), \quad C^{\prime \prime}=C \cap \prod_{1}\left(P_{i} \mid i \in I\right) .
$$

We prove that $\left|C^{\prime}\right|<\mathfrak{m}$; it follows in a dual manner that $\left|C^{\prime \prime}\right|<\mathfrak{m}$, and so $|C|<\mathfrak{m}$.

Let

$$
C_{n}^{\prime}=\left\{x \mid x \in C^{\prime} \text { and }\left|\operatorname{sp}_{0}(x)\right|=n\right\} \text {. }
$$

We observe that if $x, y \in \prod_{0}\left(P_{i} \mid i \in I\right)$ and $x \leqq y$ then $\mathrm{sp}_{0} x \subseteq \operatorname{sp}_{0} y$; thus if $x \leqq y$ and $\left|\mathrm{sp}_{0}(x)\right|=\left|\mathrm{sp}_{0}(y)\right|$ then $\mathrm{sp}_{0}(x)=\mathrm{sp}_{0}(y)$. Thus $\mathrm{sp}_{0}(x), x \in C_{n}^{\prime}$, is independent of $x$; denote this subset of $I$ by $\operatorname{sp}_{0}\left(C_{n}^{\prime}\right)$. For each $x \in \operatorname{sp}_{0}\left(C_{n}^{\prime}\right),\left\{x_{i} \mid x \in C_{n}^{\prime}\right\}$ is a chain in $P_{\mathfrak{i}}$ and so has cardinality $<\mathfrak{m}$. Thus $\left|C_{n}^{\prime}\right|<\mathfrak{m}^{n}=\mathfrak{m}$. Since $C^{\prime}=\bigcup\left(C_{n}^{\prime} \mid n<\omega\right)$ and $\mathfrak{m}$ is not cofinal with $\omega,\left|C^{\prime}\right|<\mathfrak{m}$. Thus the lemma is established.

Let $C$ be a chain with 0 and 1 . Let $B(C)$ denote the set of subsets of $C$ of the form

$$
\left(a_{0}, b_{0}\right] \cup\left(a_{1}, b_{1}\right] \cup \cdots \cup\left(a_{n-1}, b_{n-1}\right]
$$

where $a_{0}<b_{0}<a_{1}<\cdots<a_{n-1}<b_{n-1}$, and the set $\varnothing$. Identify $a \in C$ with $(0, a]$. Set inclusion makes $B(C)$ into a Boolean algebra, $C \subseteq B(C),[C]=B(C)$. Also, $|B(C)|=|C|$ if $|C| \geqq \aleph_{0} . B(C)$ is called the Boolean algebra generated by $C$. It has a chain of cardinality $|C|$, but no chain of cardinality $>|C|$.

Lemma 7. Let $\mathfrak{m}$ be an infinite cardinal not cofinal with $\omega$. There is a Boolean algebra $B$ such that

(i) B satisfies the $\mathfrak{m}$-chain condition;

(ii) for each $\mathfrak{m}_{1}<\mathfrak{m}$ there is a chain in $B$ of cardinality $\mathfrak{m}_{1}$.

Proof. Let $\left(\mathfrak{m}_{i} \mid i \in I\right)$ be the set of all infinite cardinals $<\mathfrak{m}$. For each $i \in I$ let $X_{i}$ be a chain of cardinality $\mathfrak{m}_{\mathfrak{i}}$, and let $B_{i}$ be the Boolean algebra generated by $X_{i}$. Thus for each $i \in I, B_{\mathfrak{i}}$ satisfies the $m$-chain condition and has a chain of cardinality $\mathfrak{m}_{i} . B=\prod_{w}\left(B_{i} \mid i \in I\right)$. Then $B$ is a Boolean algebra and, by Lemma $6, B$ satisfies conditions (i) and (ii).

THEOREM 5. Let $\mathfrak{m}$ be singular and not cofinal with $\omega$. Then there are Boolean algebras $B_{0}, B_{1}$ satisfying the m-chain condition such that their free product does not satisfy the $\mathrm{m}$-chain condition. 
Proof. Since $\mathfrak{m}$ is singular and not cofinal with $\omega$, there is an ordinal $\alpha, \bar{\alpha}<\mathfrak{m}$, $\alpha>\omega$, and a family of cardinals $\left(\mathfrak{m}_{\beta} \mid \beta<\alpha\right)$ such that

(a) $\mathfrak{m}_{\beta}<\mathfrak{m}$ for all $\beta<\alpha$;

(b) $\sum\left(\mathfrak{m}_{\beta} \mid \beta<\alpha\right)=\mathfrak{m}$.

Let $P=\{\beta \mid \beta \leqq \alpha\}$ and let $B_{0}$ be the Boolean algebra generated by $P$. Then $B_{0}$ satisfies the $\mathfrak{m}$-chain condition. Let $B_{1}$ be a Boolean algebra satisfying the conditions of Lemma 7 . Let $B$ be the $\{0,1\}$-distributive free product of $B_{0}$ and $B_{1}$. As mentioned in the introduction, $B$ is the free product of $B_{0}$ and $B_{1}$ in the category of Boolean algebras. For each $\beta<\alpha$ let $C_{\beta}$ be a chain in $B_{1}-\{0,1\}$ of cardinality $\mathfrak{m}_{\beta}$. Let $C \subseteq B$ be defined by

$$
C=\left\{\left(\beta^{+} \wedge y\right) \vee \beta \mid \beta<\alpha \text { and } y \in C_{\beta}\right\} .
$$

$\left(\beta^{+}\right.$denotes the successor ordinal of $\beta$.) We observe that $\left\{\left\{\beta^{+}, y\right\},\{\beta\}\right\}$ is a normal $\bigvee$-representation and that $C$ is a chain in $B$. By the uniqueness of normal $\bigvee$ representations,

$$
|C|=\sum\left(\mathfrak{m}_{\beta} \mid \beta<\alpha\right)=\mathfrak{m} .
$$

Thus the theorem is proved.

Therefore, if $\mathfrak{m}$ is singular and not cofinal with $\omega$ then $P(\mathfrak{m}, 2)$ fails to hold in the category of Boolean algebras.

If $\mathfrak{m}$ is a cardinal cofinal with $\omega$ we show in a similar manner that $P\left(\mathfrak{m}, \boldsymbol{N}_{0}\right)$ fails to hold for Boolean algebras:

THEOREM 6. Let $\mathfrak{m}$ be a cardinal cofinal with $\omega$. There is a set I of cardinality $\boldsymbol{\aleph}_{0}$ and a family $\left(B_{i} \mid i \in I\right)$ of Boolean algebras satisfying the $\mathfrak{m}$-chain condition such that their free product $B$ does not satisfy the $\mathfrak{m}$-chain condition.

Proof. If $\mathfrak{m}=\boldsymbol{\aleph}_{0}$ take countably many finite Boolean algebras with more than one element.

If $\mathfrak{m} \neq \boldsymbol{\aleph}_{0}$ then there is a family of infinite cardinals $\left(\mathfrak{m}_{\mathfrak{i}} \mid i<\omega\right)$ such that $\mathfrak{m}_{\mathfrak{i}}<\mathfrak{m}$ for all $i<\omega$ and $\mathfrak{m}=\sum\left(\mathfrak{m}_{i} \mid i<\omega\right)$. Let $I=\{i \mid i \leqq \omega\}$. For each $i<\omega$ let $B_{i}$ be the Boolean algebra generated by a chain of cardinality $\mathfrak{m}_{i}$. Then $B_{i}$ satisfies the $\mathfrak{m}$ chain condition and $B_{i}-\{0,1\}$ contains a chain $C_{i}$ of cardinality $\mathfrak{m}_{\mathfrak{i}}$. Let $B_{\omega}$ be the Boolean algebra generated by the chain $\{\alpha \mid \alpha \leqq \omega\}$; since $\mathfrak{m}>\boldsymbol{\aleph}_{0}, B_{\omega}$ satisfies the $m$-chain condition. Then, as in Theorem 5 ,

$$
C=\left\{(y \vee(i+1)) \wedge i \mid y \in C_{i}, i<\omega\right\}
$$

is a chain in the free product of the $\left(B_{i} \mid i \in I\right)$ of cardinality $m$.

To resolve the remaining case we first establish the following lemma.

LEMMA 8. Let $\mathfrak{m}$ be cofinal with $\omega$ and let $L$ be a distributive lattice that has a chain of cardinality $\mathfrak{m}_{1}$ for each $\mathfrak{m}_{1}<\mathfrak{m}$. Then $L$ has a chain of cardinality $\mathfrak{m}\left({ }^{6}\right)$.

$\left.{ }^{(}\right)$The authors express their appreciation to A. Hajnal for communicating the statement and the proof of this lemma to them. 
Proof. If $\mathfrak{m}=\boldsymbol{\aleph}_{0}$, then $L$ has an infinite chain since $L$ is infinite.

If $\mathfrak{m}>\mathcal{N}_{0}$ then there is a sequence $(\mathfrak{m}(i) \mid i<\omega)$ of infinite cardinals such that

(i) if $i<j$ then $\mathfrak{m}(i)<\mathfrak{m}(j)<\mathfrak{m}$;

(ii) for each $\mathfrak{m}^{\prime}<\mathfrak{m}$ there is an $i<\omega$ such that $\mathfrak{m}^{\prime}<\mathfrak{m}(i)$.

We can assume that $L$ has a 1 . There are two cases to consider.

Case 1. There is an isotone mapping $f:[0, \omega) \rightarrow[0, \omega)$ such that for each $i<\omega$ there is an $a(i) \in L$ containing a chain of cardinality $\mathfrak{m}(i)$ but no chain of cardinality $\mathfrak{m}(f(i))$.

For each $i<\omega$ let $b_{i}=a\left(f^{i}(0)\right), \mathfrak{n}_{i}=\mathfrak{m}\left(f^{i}(0)\right)$. (Note that $\mathfrak{n}_{i} \geqq \mathfrak{m}(i)$.) Thus, for each $i<\omega,\left(b_{i}\right]$ contains a chain $C_{\mathfrak{i}}$ of cardinality $\mathfrak{n}_{\mathfrak{i}}$, but no chain of cardinality $\mathfrak{n}_{\mathfrak{i}+1}$. For each $i<\omega$ let

$$
D_{i}=\left\{b_{0} \vee \cdots \vee b_{i-1} \vee x \mid x \in C_{i}\right\}
$$

Then $C=\bigcup\left(D_{i} \mid i<\omega\right)$ is a chain in $L$. To show that $|C|=\mathfrak{m}$ we need only show that $\left|D_{i}\right|=\mathfrak{n}_{\mathfrak{i}}$.

For each $i<\omega$ there is a 1-1 lattice homomorphism

$$
\left(b_{0} \vee \cdots \vee b_{i-1}\right] \rightarrow\left(b_{0}\right] \times \cdots \times\left(b_{i-1}\right]
$$

and so, since the sequence $\left\{\mathfrak{n}_{\mathfrak{i}}\right\}$ is strictly increasing, $\left(b_{0} \vee \cdots \vee b_{i-1}\right]$ contains no chain of cardinality $\mathfrak{n}_{\mathfrak{h}}$.

We observe that there is a 1-1 lattice homomorphism

$$
\left(b_{i}\right] \rightarrow\left(\left(b_{0} \vee \cdots \vee b_{i-1}\right) \wedge b_{i}\right] \times\left[\left(b_{0} \vee \cdots \vee b_{i-1}\right) \wedge b_{i}, b_{i}\right]
$$

which, composed with the isomorphism

$$
\left[\left(b_{0} \vee \cdots \vee b_{i-1}\right) \wedge b_{i}, b_{i}\right] \cong\left[b_{0} \vee \cdots \vee b_{i-1}, b_{0} \vee \cdots \vee b_{i}\right],
$$

embeds $C_{i}$ in $C_{i}^{\prime} \times D_{i}$, where $C_{i}^{\prime}$ is a chain in

$$
\left(\left(b_{0} \vee \cdots \vee b_{i-1}\right) \wedge b_{i}\right] \subseteq\left(b_{0} \vee \cdots \vee b_{i-1}\right] .
$$

Since $\left|C_{\mathfrak{i}}^{\prime}\right|<\mathfrak{n}_{\mathfrak{i}}$ and $\left|C_{\mathfrak{i}}\right|=\mathfrak{n}_{\mathfrak{i}}$ we conclude that $\left|D_{\boldsymbol{i}}\right|=\mathfrak{n}_{\mathfrak{i}}$.

Case 2. There is an $n<\omega$ such that if $a \in L$ and (a] contains a chain of cardinality $\mathfrak{m}(n)$ then $(a]$ contains a chain of cardinality $\mathfrak{m}(i)$ for all $i>n$.

We construct a chain of cardinality $\mathfrak{m}$ in $L$. Let $a_{0}=1$; then $\left(a_{0}\right]$ contains a chain $C_{0}$ of cardinality $\mathfrak{m}(n+1)$. Since $\mathfrak{m}(n)<\mathfrak{m}(n+1)$ there is an element $a_{1} \in C_{0}$ such that the chains $D_{0}=\left[a_{1}\right) \cap C_{0}$ and $\left(a_{1}\right] \cap C_{0}$ each have cardinality $\geqq \mathfrak{m}(n)$. Thus $\left(a_{1}\right]$ contains a chain $C_{1}$ of cardinality $\mathfrak{m}(n+2)$ and we find $a_{2} \in C_{1}$ such that the chains $D_{1}=\left[a_{2}\right) \cap C_{1}$ and $\left(a_{2}\right] \cap C_{1}$ each have cardinality $\geqq \mathfrak{m}(n+1)$.

Proceeding inductively we find elements $a_{i+1}$ and chains $C_{i}, D_{i}$ such that

(i) $\left|D_{i}\right| \geqq \mathfrak{m}(n+i)$;

(ii) if $x \in D_{i+1}, y \in D_{i}$ then $x \leqq y$.

Thus $D=\bigcup\left(D_{i} \mid i<\omega\right)$ is a chain of cardinality $m$, concluding the proof of this lemma. 
THEOREM 7. Let I be a finite set and let $\mathfrak{m}$ be a cardinal cofinal with $\omega$. If $\left(L_{i} \mid i \in I\right)$ is a family of distributive lattices with 0,1 such that $L_{i}$ satisfies the $m$-chain condition for each $i \in I$ then the $\{0,1\}$-distributive free product of the $\left(L_{i} \mid i \in I\right)$ satisfies the $\mathfrak{m}$-chain condition.

Proof. If $\mathfrak{m}=\boldsymbol{\aleph}_{0}$, then each $L_{i}, i \in I$, is finite, (since an infinite distributive lattice contains an infinite chain) and thus their $\{0,1\}$-distributive free product is finite.

If $\mathfrak{m}>\boldsymbol{N}_{0}$ then, by Lemma 8 , there is an infinite cardinal $\mathfrak{m}_{i}<\mathfrak{m}$ for each $i \in I$ such that $L_{i}$ satisfies the $\mathfrak{m}_{i}$-chain condition for each $i \in I$. Let $\mathfrak{m}^{\prime}=\max \left(m_{i}^{+} \mid i \in I\right)$. Since $I$ is finite $\mathfrak{m}^{\prime}$ is a successor cardinal and thus $\mathfrak{m}^{\prime}$ is regular. Since $L_{\mathfrak{i}}$, for each $i \in I$, satisfies the $\mathfrak{m}^{\prime}$-chain condition, so does the free product; thus $\mathfrak{m}^{\prime} \leqq \mathfrak{m}$ implies that the free product satisfies the $m$-chain condition.

We observe that in any category of lattices $P(\mathfrak{m}, \mathfrak{n})$ implies $P\left(\mathfrak{m}, \mathfrak{n}_{1}\right)$ for all $\mathfrak{n}_{1} \leqq \mathfrak{n}$. Thus, in view of the facts presented in the introduction concerning the relation between the three categories, Theorems 4, 5, 6 and 7 combine to yield our main result:

THEOREM. For each of the category of distributive lattices, the category of distributive lattices with 0,1 , and the category of Boolean algebras:

(a) if $\mathfrak{n} \geqq \aleph_{0}$ then $P(\mathfrak{m}, \mathfrak{n})$ holds if and only if $\mathfrak{m}$ is regular and greater than $\boldsymbol{\aleph}_{0}$;

(b) if $1<\mathfrak{n}<\aleph_{0}$ then $P(\mathfrak{m}, \mathfrak{n})$ holds if and only if $\mathfrak{m}$ is either regular or cofinal with $\omega$.

\section{BIBLIOGRAPHY}

1. F. Galvin and B. Jónsson, Distributive sublattices of a free lattice, Canad. J. Math. 13 (1961), 265-272.

2. G. Grätzer, Universal algebra, Van Nostrand, Princeton, N. J., 1968.

3. A. Horn, A property of free Boolean algebras, Proc. Amer. Math. Soc. 19 (1968), 142-143.

4. A. Nerode, Some Stone spaces and recursion theory, Duke Math. J. 26 (1959), 397-406.

5. I. Reznikoff, Chaines de formules, C. R. Acad. Sci. Paris 256 (1963), 5021-5023.

UNIVERSITY OF MANITOBA,

Winnipeg, Manitoba, Canada 